\title{
A Consideration of Magnetostriction Force Calculation for Transformer Core by Using Reluctance Network Analysis
}

\author{
Y. Hane, K. Nakamura, and N. Kurita* \\ Graduate School of Engineering, Tohoku Univ., 6-6-11 Aoba Aramaki, Aoba-ku, Sendai 980-8579, Japan \\ ${ }^{*}$ Hitachi, Ltd., 7-1-1 Omikacho, Hitachi 319-1292, Japan
}

In recent years, it has been strongly required to reduce radiated noises from transformer cores used in electric power systems. The noises from transformer cores seem to be mainly caused by magnetostriction. Thus, it is necessary to establish a method for quantitatively calculating the force generated from the magnetostriction (magnetostriction force) with not only high-accuracy but also high-speed. This paper presents that the magnetostriction force is calculated based on a reluctance network analysis (RNA) by using a simple-shape wound core as an object of discussion. The validity of the proposed method is indicated by comparing with calculation results obtained from a finite element method (FEM).

Key words: finite element method (FEM), magnetostriction force, reluctance network analysis (RNA)

\section{Introduction}

In recent years, it is strongly required to reduce noises radiated from transformers used in electric power systems. The causes of a noise of electric machines are mainly divided into the electromagnetic force and the magnetostriction generated in an iron core. Among them, the magnetostriction is considered to be the major cause of the noise for transformers ${ }^{1}$. Therefore, in order to reduce the acoustic noise due to the magnetostriction for transformers, it is necessary to establish a method for calculating the force generated from the magnetostriction (magnetostriction force). However, it is difficult to theoretically analyze the magnetostriction such as based on micromagnetics. To overcome this problem, Kitagawa et al. ${ }^{2)}$ presented the method which calculates the magnetostriction force by using the magnetic flux density distribution obtained from a finite element method (FEM), and the magnetic flux density versus magnetostriction characteristics of the core material obtained from the measurement.

However, in order to design and analyze transformers for the purpose of acoustic noise reduction, it is essential to calculate displacement of a shape of an iron core by structural analysis, and the radiated sound by acoustic analysis, based on the magnetostriction force obtained from electromagnetic field analysis. It leads the analytical model to be very large, which requires a long calculation time and a huge computer capacity. Therefore, in order to realize electromagneticstructural-acoustic coupled analysis, the analytical model should be simple as well as with high calculation accuracy.

To solve the above problem, this paper presents that the calculation method of the magnetostriction force proposed in the reference 2) is applied to a reluctance

Corresponding author: Y. Hane (e-mail: yoshiki.hane @ecei.tohoku.ac.jp). network analysis (RNA). The RNA expresses an analytical object by one reluctance network. All the reluctances can be determined by $B-H$ curve of the material and dimensions 3). The RNA has some advantages such as a simple model, fast calculation, and easy coupling with external electric circuits and motion equation. The RNA has been applied to the calculation of characteristics of various electric machines including transformers and motors ${ }^{4)-6)}$. In this paper, the validity of the proposed method is proved by comparing with the calculation results obtained from a two-dimensional (2-D) FEM.

\section{Reluctance Network Analysis}

In this chapter, a method for deriving a 2-D RNA model of a wound core made of an amorphous alloy, which is generally used for transformers, is explained.

First, the analytical object is divided into multiple elements as shown in Fig. 1(a). Here, the core is divided into 3 in the thickness direction and 16 in the rolling direction, respectively. Each divided element can be expressed in a 2-D unit magnetic circuit as shown in Fig. 1(b). Each reluctance can be determined in consideration of the nonlinear magnetic characteristics. Here, the magnetic nonlinearity is given by

$$
H=\alpha_{1} B+\alpha_{m} B^{m}
$$

where the coefficients are $\alpha_{1}=2.21$ and $\alpha_{m}=4.88 \times 10^{-4}$, respectively. The order $m=15$ is determined by the strength of the nonlinearity of the $B-H$ curve. Fig. 2 shows the measured $B-H$ curve of the core material and its approximated curve.

From the equation (1), the relationship between the magnetomotive force (MMF) $f_{m}$ and the magnetic flux $\phi$ in each reluctance can be expressed by the following equation:

$$
f_{m}=\left(\frac{\alpha_{1} l}{S}+\frac{\alpha_{m} l}{S^{m}} \phi^{m-1}\right) \phi
$$




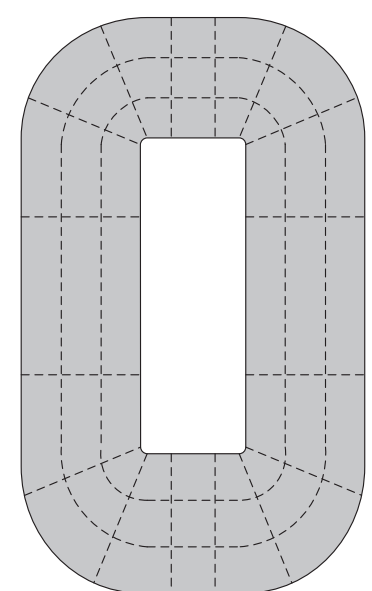

(a) Division of a wound core

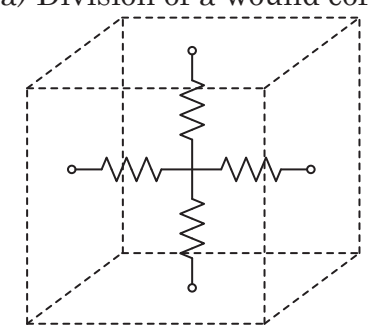

(b) Unit magnetic circuit.

Fig. 1 Division of a wound core based on RNA.

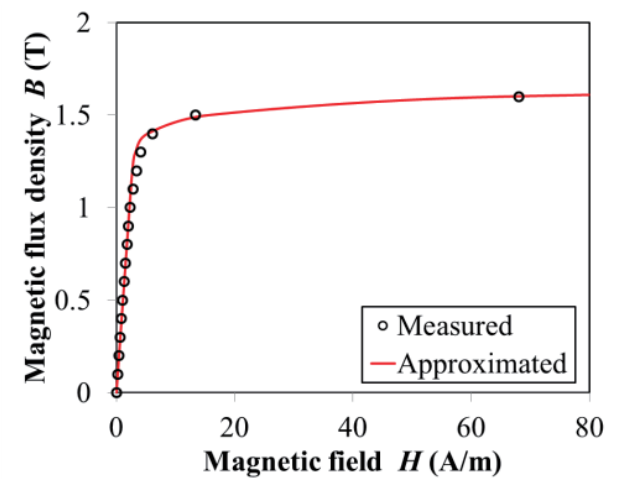

Fig. $2 \quad B$ - $H$ curve of core material and its approximated curve.

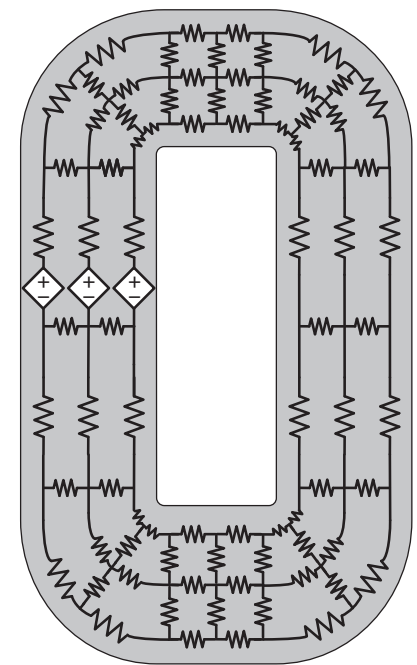

Fig. 3 Schematic diagram of a 2-D RNA model of a wound core. where the average cross-sectional area and magnetic path length of each element are $S$ and $l$, respectively. Therefore, the nonlinear reluctance $R_{m}$ is given by the following equation:

$$
R_{m}=\frac{\alpha_{1} l}{S}+\frac{\alpha_{m} l}{S^{m}} \phi^{m-1}
$$

Here, it is assumed that the magnetic properties of the core are isotropic for simplicity.

Fig. 3 shows a schematic diagram of the derived 2-D RNA model of the wound core. The MMFs are generated from the winding current.

\section{Calculation Method for Magnetostriction Force}

As mentioned above, it is difficult to theoretically calculate the magnetostriction force. Therefore, in this paper, the magnetostriction force is calculated by using the measured values of the magnetic flux density versus magnetostriction characteristics of the core material, based on the method proposed in the reference 2). The magnetic flux distribution of the core can be obtained from the RNA. In this chapter, first, the magnetic flux density versus magnetostriction characteristics used for the analysis are shown. Next, the calculation method of the magnetostriction force for the RNA is described.

\subsection{Magnetic flux density versus magnetostriction characteristics}

In general, it is difficult to measure the magnetostriction from the amorphous alloy because its thickness is very thin. Thus, in this paper, the alternative data is created by correcting the peak-to-peak value of the magnetostriction curve of the grain-oriented silicon steel 7 ) to $27 \mathrm{ppm}$ which is nominal value of the magnetostriction of the amorphous alloy. Fig. 4 shows the corrected magnetic flux density versus magnetostriction characteristics used as a look-up table in the analysis.

\subsection{Calculation method for magnetostriction force}

The magnetostriction force applied to the edges of each element is expressed by the following equation, based on the Hooke's law.

$$
\boldsymbol{p}=\int_{S} \boldsymbol{D} \boldsymbol{\varepsilon} d S
$$

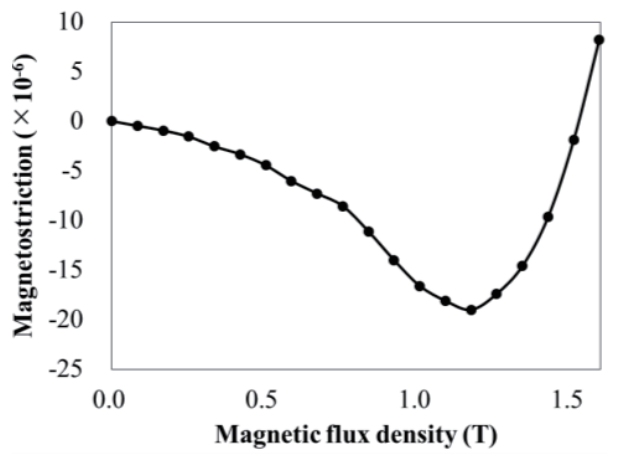

Fig. 4 Magnetic flux density versus magnetostriction characteristics. 
where $\boldsymbol{p}$ is the magnetostriction force, which is expressed by the following equation when the shear force is neglected:

$$
\boldsymbol{p}=\left[\begin{array}{lll}
p_{x} & p_{y} & 0
\end{array}\right]^{\mathrm{T}}
$$

where $p_{x}$ and $p_{y}$ are the $x$ - and $y$-axis components of $\boldsymbol{p}$, which work in the rolling and vertical directions of each element, respectively. Here, $\boldsymbol{D}$ is the elasticity matrix expressed by the following equation.

$$
\boldsymbol{D}=\frac{E}{(1+v)(1-2 v)}\left[\begin{array}{ccc}
1-v & v & 0 \\
v & 1-v & 0 \\
0 & 0 & (1-2 v) / 2
\end{array}\right]
$$

where $E$ is Young's modulus and $v$ is Poisson's ratio, respectively. Here, $E=120 \mathrm{GPa}$ and $v=0.3$, respectively. In addition, $\varepsilon$ in the equation (4) is magnetostriction, which can be expressed by the following equation when the shear strain is neglected.

$$
\boldsymbol{\varepsilon}=\left[\varepsilon_{x}\left(B_{x}, B_{y}\right) \quad \varepsilon_{y}\left(B_{x}, B_{y}\right) \quad 0\right]^{\mathrm{T}}
$$

Here, the magnetostriction $\varepsilon_{x}\left(B_{x}, B_{y}\right)$ and $\varepsilon_{y}\left(B_{x}, B_{y}\right)$ generated in the $x$ - and $y$-axis directions, that is the rolling and vertical directions, are expressed by the following equations, as the sum of strains generated by the magnetic flux densities $B_{x}$ and $B_{y}$ in the $x$ and $y$ axis directions, respectively.

$$
\begin{aligned}
& \varepsilon_{x}\left(B_{x}, B_{y}\right)=\varepsilon_{x 0}\left(B_{x}\right)-v \varepsilon_{y 0}\left(B_{y}\right) \\
& \varepsilon_{y}\left(B_{x}, B_{y}\right)=-v \varepsilon_{x 0}\left(B_{x}\right)+\varepsilon_{y 0}\left(B_{y}\right)
\end{aligned}
$$

where $\varepsilon_{x 0}$ and $\varepsilon_{y 0}$ are magnetostriction corresponding to the magnetic flux densities $B_{x}$ and $B_{y}$ in the magnetic flux density versus magnetostriction characteristics shown in Fig. 4.

\section{Simulation Results}

By using the proposed method, the magnetostriction force applied to the wound core is calculated based on the magnetic flux density distribution obtained from RNA when the sine wave voltage, with the amplitude of $100 \mathrm{~V}$ and the frequency of $50 \mathrm{~Hz}$, is applied. The validity of the proposed method is verified by comparing with the calculation results obtained from the 2-D FEM by using the JMAG-Designer Ver.18.1, which is general-purpose electro-magnetic simulation software. Fig. 5 shows the enlarged view of 2-D FEM model of the

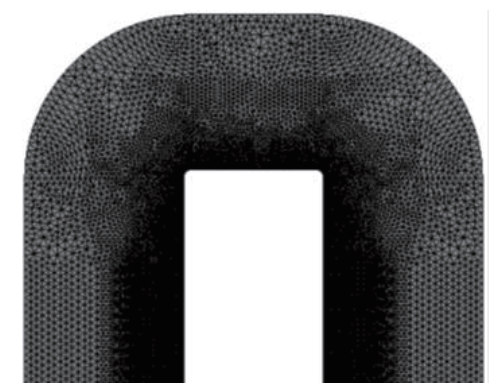

Fig. 5 Enlarged view of 2-D FEM model of a wound core. wound core, with 73,300 elements. In addition, Fig. 6 and 7 show contour maps of calculation results of magnetic flux density and magnetostriction force obtained from the FEM, respectively.

Fig. 8 shows the edges where calculation results are compared. The average magnetic flux densities of

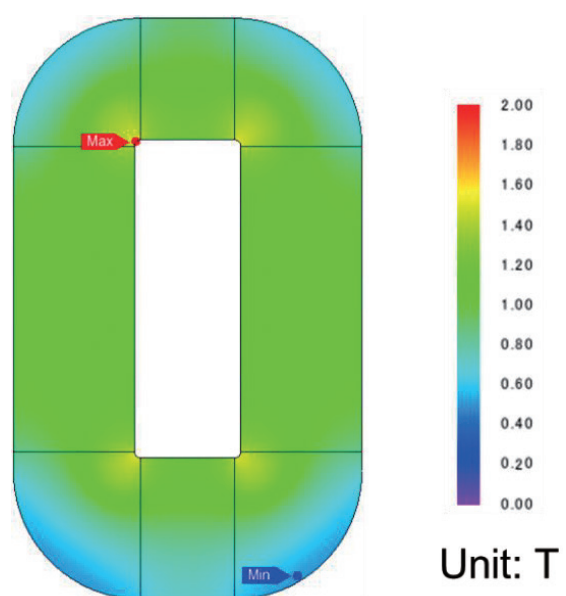

Fig. 6 Contour map of calculation result of magnetic flux density obtained from FEM.

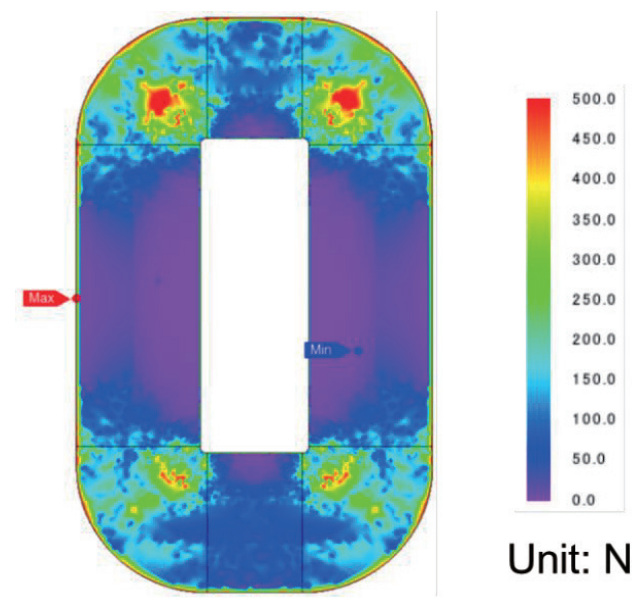

Fig. 7 Contour map of calculation result of magnetostriction force obtained from FEM.

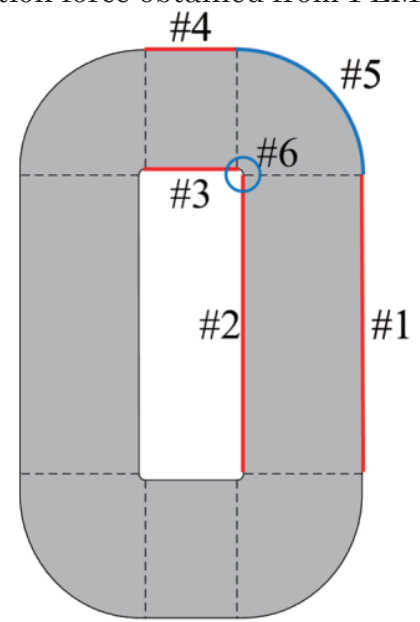

Fig. 8 Compared edges of calculation results. 


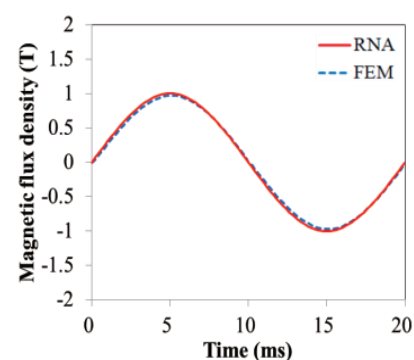

(a) Edge \#1

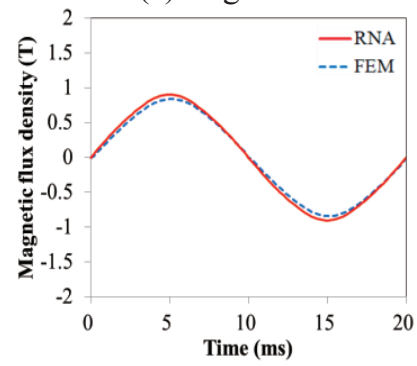

(c) Edge \#3

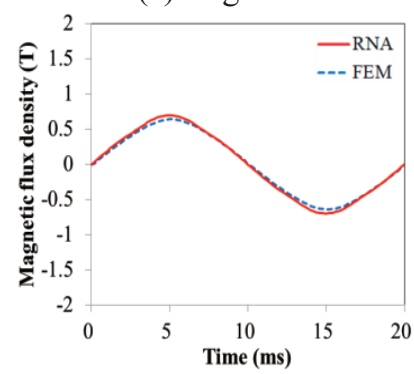

(e) Edge \#5

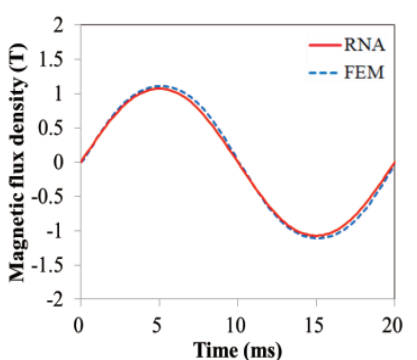

(b) Edge \#2

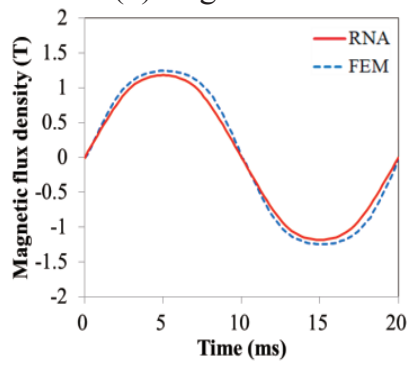

(d) Edge \#4

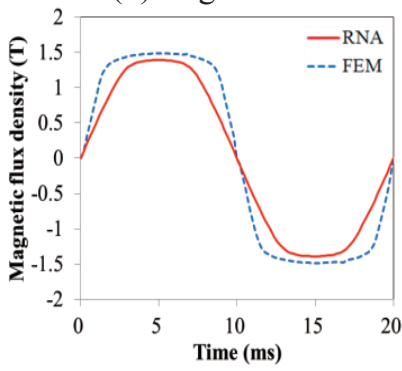

(f) Edge \#6

Fig. 9 Comparison of calculation results of magnetic flux density waveforms obtained from RNA and FEM.

elements adjacent to the edges \#1 \#6, and the magnetostriction forces applied in the direction perpendicular to the edges \#1 \#6 in the figure are compared between the RNA and the FEM.

Fig. 9(a)-(f) show the comparisons of calculation results of the magnetic flux density waveforms, which are obtained by taking the average value in the rolling direction in every element adjacent to each edge, for both the RNA and FEM. From these figures, it is understood that the calculated values of the RNA and FEM are in good agreement on the edges \#1 \#5, while there is a large error on the edge \#6.

Fig. 10(a)-(f) show the comparisons of calculation results of the magnetostriction force waveforms, which are calculated by summing the forces acting perpendicular to the edges for all elements adjacent to the corresponding edge, for both the RNA and FEM. From these figures, it is clear that the calculated values of the RNA and FEM are almost in good agreement, although the errors are larger compared with those of the magnetic flux density waveforms. On the other hand, there is a large error as well as on the edge \#6. It is assumed that one of the causes of these errors is the calculation accuracy of the magnetic flux density distribution due to the difference in the number of divided elements between the RNA and FEM.

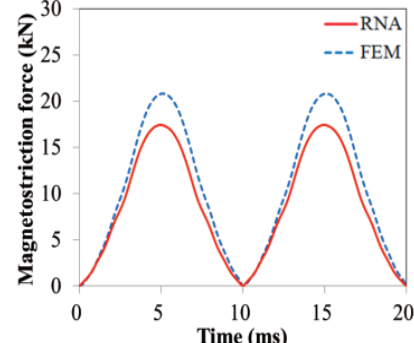

(a) Edge \#1

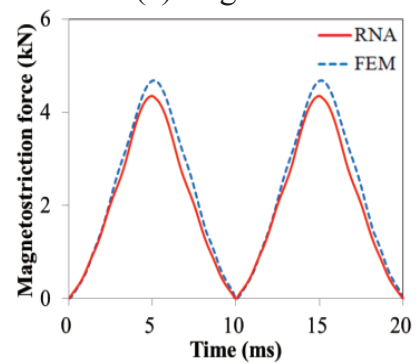

(c) Edge \#3

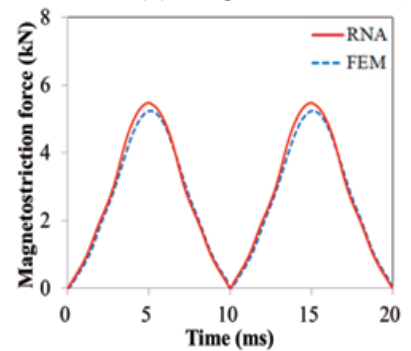

(e) Edge \#5

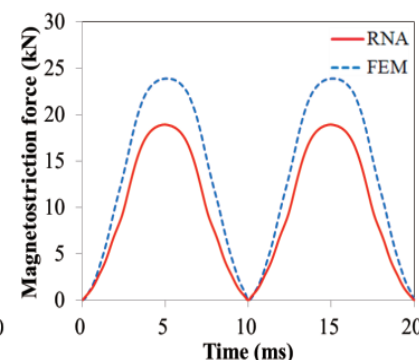

(b) Edge \#2

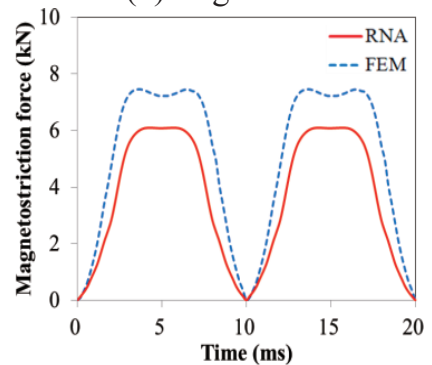

(d) Edge \#4

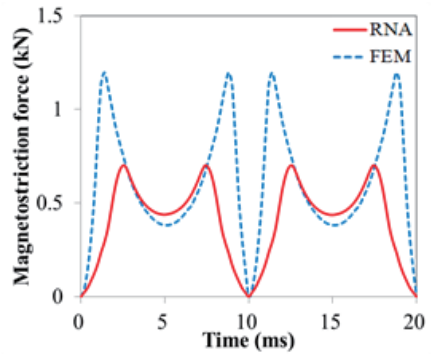

(f) Edge \#6
Fig. 10 Comparison of calculation results of magnetostriction force waveforms obtained from RNA and FEM.

Therefore, in order to verify the cause of the above errors, the magnetic flux density and magnetostriction force are calculated by using the more finely divided RNA model. Here, the core is divided into 5 by a ratio of 1:4:5:4:1 in the thickness direction, and 32 in the rolling direction, respectively. Fig. 11 shows a division of the finely divided RNA model of the wound core.

Fig. 12(a)-(f) show the comparisons of calculation results of the magnetic flux density waveforms. From these figures, it is understood that the calculated values of the RNA and FEM are in good agreement on all the edges.

Fig. 13(a)-(f) show the comparisons of calculation results of the magnetostriction force waveforms. From these figures, it is clear that the measured and calculated values are almost in good agreement on all the edges. The cause of the error seems to be that the self-made algorithm of the magnetostriction force calculation for the RNA is strictly different from JMAG algorithm.

Additionally, the calculation time is less than 1 second for the RNA whereas approximately 1 minute for the FEM, which indicates that the proposed method can significantly shorten the calculation time when the 3-D electromagnetic-structural-acoustic coupled analysis will be performed in the future. 


\section{Conclusion}

This paper presented the calculation method for the magnetostriction force based on the RNA.

As a result, it was clear that the proposed method can calculate the magnetostriction force with relatively high accuracy and in a very short time, compared with the results of the FEM.

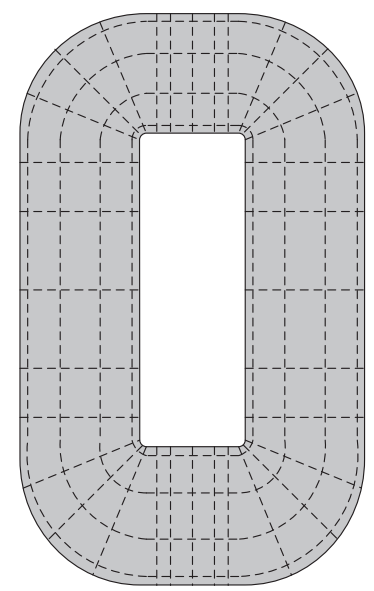

Fig. 11 Division of a finely divided RNA model of a wound core.

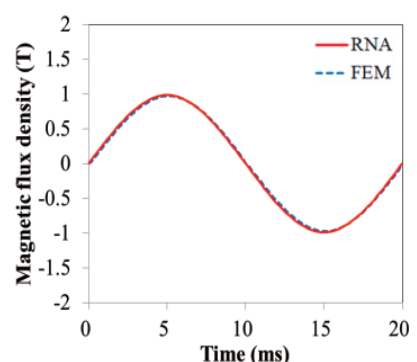

(a) Edge \#1

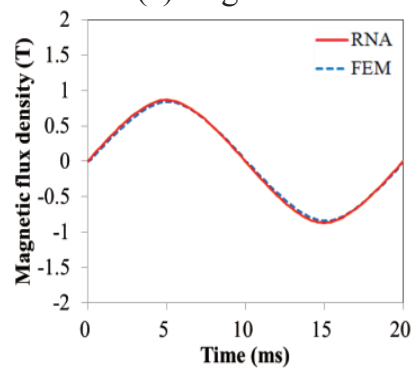

(c) Edge \#3

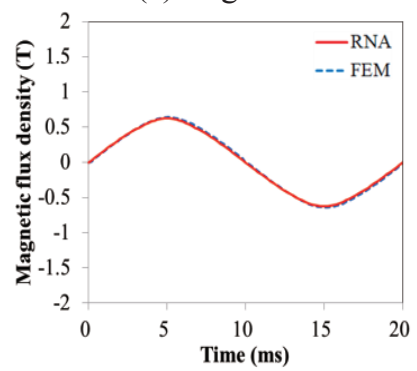

(e) Edge \#5

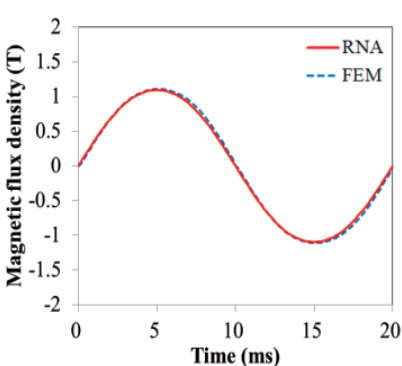

(b) Edge \#2

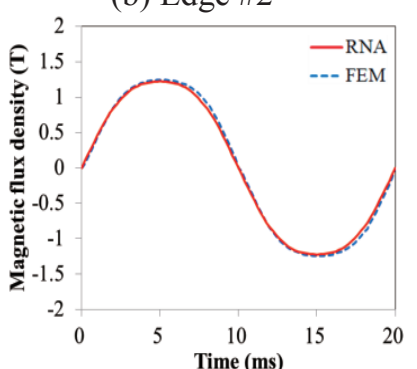

(d) Edge \#4

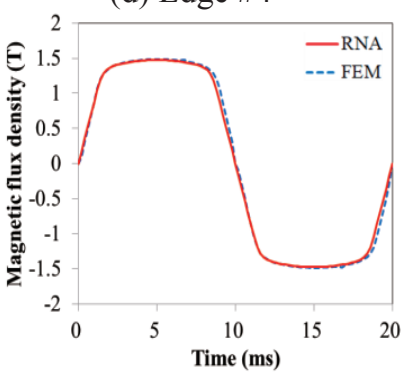

(f) Edge \#6
Fig. 12 Comparison of calculation results of magnetic flux density waveforms obtained from finely divided RNA and FEM.

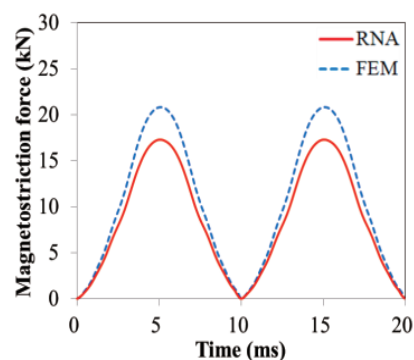

(a) Edge \#1

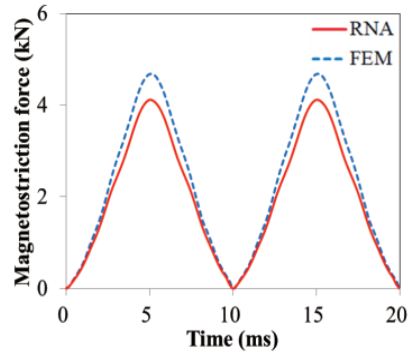

(c) Edge \#3

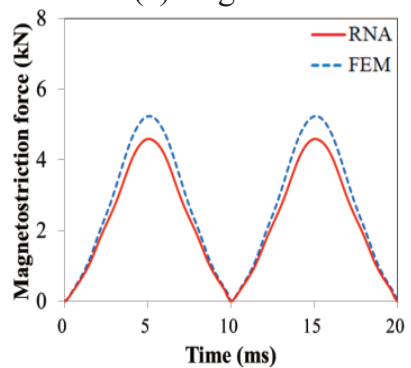

(e) Edge \#5

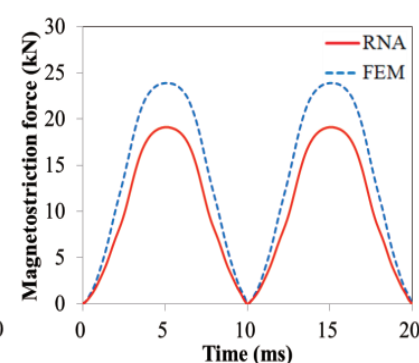

(b) Edge \#2

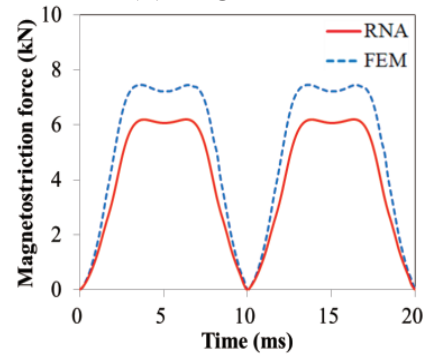

(d) Edge \#4

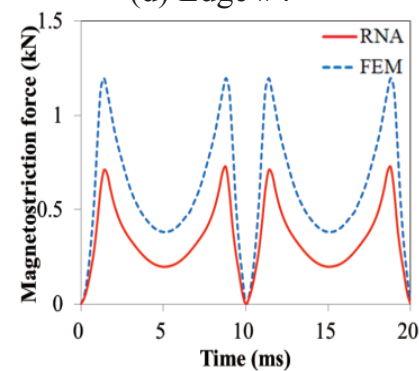

(f) Edge \#6
Fig. 13 Comparison of calculation results of magnetostriction force waveforms obtained from finely divided RNA and FEM.

In the future, we plan to perform structural and acoustic analysis by using the obtained magnetostriction force, and prove the validity of the proposed method experimentally.

Acknowledgements This work was supported by Grant-in Aid for JSPS Fellows (JP19J20572).

\section{References}

1) A. J. Moses, IEEE Trans. Magn., 10, 154 (1974).

2) W. Kitagawa, Y. Ishihara, T. Todaka, and A. Nakasaka, IEEJ Trans. PE, 128, 654 (2008).

3) K. Nakamura and O. Ichinokura, IEEJ Trans. FM, 128, 506 (2008).

4) K. Nakamura, K. Kimura, and O. Ichinokura, Journal of Magnetism and Magnetic Materials, 290-291, 1309 (2005).

5) M. Fukuoka, K. Nakamura, and O. Ichinokura, IEEE Trans. Magnetics, 47, 2414 (2011).

6) K. Nakamura, K. Honma, T. Ohinata, K. Arimatsu, T. Shirasaki, and O. Ichinokura, Journal of the Magnetics Society of Japan, 38, 174 (2014)

7) S. Taguchi, T. Yamamoto, and A. Sakakura, IEEE Trans. Magn., 10, 123 (1974).

Received Dec. 23, 2020; Revised Feb. 2, 2021; Accepted Feb. 10,2021 